\title{
Resumen de Revistas
}

IIIPTURA DE HERIDA DE MIOMECTOMIA DURANTE EL EMBA. RAZO-Mackie J. E.-J. Obst. Gynaec. Brit. Emp., 59: 838, Dic. 1952. El autor, de la Universidad de Edimburgo y del Simpson Memorial Maternity.

La ruptura del útero gestante posterior a miomectomía es un desastre poco común. El autor dice que no se había notado esta complicación en el Simpson Mcm. Mat. Hosp. en los últimos 20 años, sobre un total de 50 casos de ruptura utcrina durante el embarazo. Quizás el único caso reportado anteriormente en la litcratura británica fue descrito por Gemmell en 1936.

El autor presenta un caso de una mujer de 30 años y hace un detallado M.to de la historia.

En la discusión del caso, el autor recuerda que los libros de Obstetricia mencionan frecuentemente la miomectomía como factor de ruptura uterina subwcuente. La miomectomía es una operación muy común y se computa que la icrerra parte de estas pacientes quedan luego embarazadas.

Ahltorp en 1946 revisando literatura, encuentra tan sólo 20 casos de rupturas de heridas de miomectomías en 1.300 operadas; Finn y Muller (1950) refwortin 80 embarazos en 53 pacientes sometidas previamente a miomectomías, sin una sola ruptura.

Se pregunta el autor: ¿Se debe aconsejar a la paciente con miomectomía - victor un año para buscar el embarazo? Dice: Esta espera es a menudo acon - hilke, pero se han visto muchos casos de embarazo en el primer ano de miomectomía sin complicaciones; y no olvidar que muchas de estas mujeres por su cdicl, etc., no pueden esperar mucho; en estos casos se puede permitir, con especiales cuidados prenatales, y estando listo para una cesárea.

H. A. L.

DEFORMIDADES DEL TRACTO URINARIO Y AUSENCIA CONGENITA dE la vagina.-Phelan J. T., Counseller V. S., Greene L. P.-Surg. Gyn. Obst., 97:1, julio, 1953.

Los autores de la Mayo Clinic, Rochester, Minn.

La ausencia congénita de la vagina varia entre el 1 por 5.000 (Engstad) y d I por 100.000 (Owens). Uno de los autores (Counscller) llama la atención wobre la importancia del estudio del tracto urinario de estas pacientes antes de intentar correcciones quirúrgicas, teniendo en cuenta el origen embriológico común. 
Los autores estudian 129 casos de agenesia vaginal, dividiéndolıs en 2 grupos: 1.-Con estudio urológilco: Urografía y en algunos casos, cistoscopia (72 casos); II.-Sin estudio urológico ( 57 casos). Algunas anomalias de! tracto urinario de éste último grupo fueron vistas en el examen clínico o durante la intervención operatoria.

Resultados de la investigación urológica:

I.-Anomalías significativas:

a) Urográficas: Evidencia de anormalidades se observó en 26 de las 72 pacientes. Entre las principales: Agenesia renal derecha, 4 casos; agenesia renal izquierda en 6; ectopía renal derecha en 3; ectopía izquierda en 2; ectopia bilateral en 1; riñón fusionado unilateral en 1 caso; obstrucción uretero pélvica en ambos riñones, I caso.

b) Cistoscópicas: en las practicadas en 14 de 18 pacientes con anomalias significativas; en 3 en quienes la urografía demostró agenesia renal, se vio la ausencia del correspondiente orificio ureteral; en 3 más se vio: en 1, mucosa vesical hiperplásica, y en las otras 2, defectos del piso de la uretra; en éstas ya se había intentado, sin éxito, reconstrucción quirúrgica de la vagina.

II.-Anomalías menores congénitas: Obstrucción leve de la unión uréteropélvica y malrotación de los riñones en los 8 casos restantes de los 26 anotados.

De esto se deduce la importancia del estudio urológico en esta clase de pacientes antes de someterlas a intervención quirúrgica, tanto por las complicaciones inmediatas como por las tardías.

\section{H. A. L.}

\section{REDUCCION DEL TITULO DE ANTICUERPOS ANTI R-H POR LA CORTIZONA ADMINISTRADA DUIRANTE EL EMBARAZO.- Abraham M. Frumin, M. D., Ann Gray Taylon, M. D., Eleanor Mallay Smith, M. D., Mary B. Dratman, M. D., Philadelphia, P. A. Am. J. Obst, and Ginec. June, 1953.}

Todo intento para modificar el curso de la anemia hemolítica del recién nacido debida a la incompatibilidad $\mathrm{Rh}$ ha fallado hasta hoy; los autores teniendo en cuenta la efectividad de la cortisona en la anemia hemolítica adquirida, resolvieron utilizar ésta en su tratamiento.

Doerner la halló de poca utilidad administrada durante los dos últimos meses del embarazo; sin embargo los autores después del éxito completo obtenido en una embarazada con anemia hemolítica adquirida, tratada durante todo el embarazo con cortisona y corticotropina; se resolvieron a tratar una enferma desde el sexto mes y con un elevado título de anticuerpos $\mathrm{Rh}$, obteniendo los datos siguientes: Enferma de color, de 26 años, grávida IIl para 1I, con tres meses de embarazo; primer embarazo con parto espontáneo normal; segundo hijo muerto cuatro horas después del nacimiento por una anemia hemolítica. $\mathrm{Al}$ examen de tipo, la sangre del padre dio: Al CDE/CDE; la madre resultó: Al cde/cde. La titulación de anticuerpos dio 1:512 en albúmina y 1:64 en solución salina. Se inició el tratamiento con un promedio diario de cortisona de 50 mgrs, durante 6 meses, apreciándose un descenso marcado de la titulación 
en solución salina hasta desaparecer completamente al cabo de tres meses de tratamiento; notóse igualmente que la titulación en albúmina disminuyó, pero no se logró que llegara a 0 , siendo ésta en el momento del parto de 1:32. La prueba indirecta de Coombs se sostuvo casi inmodificada, pues de 1:64 al iniciar el tratamiento, solamente bajó a $1: 128$ antes del parto.

El feto pesó 5 libras 9 onzas; mucosas pálidas, ictericia subconjuntival que se establece muy precozmente; higado y bazo palpables a nivel del ombligo; no cristia derrame ascítico. Peso de la placenta 455 gramos, ligeramente edemosa. I a dittos hematológicos obtenidos con sangre del cordón son: hematies: 1.540; licmeglobina: $7.7 \mathrm{gm}$. (50\%); reticulocitos: 99\%; leucocitos: 12.800; cọn 8\% 1. metamiclocitos, $4 \%$ de células segmentadas; $88 \%$ de linfocitos y 950 hemaiie: nucleados, por cada 100 leucocitos. La prueba de Coombs fue positiva. Bilirrubina directa (en el cordón): 3 mgrs. y la indirecta: 6,1 mgrs.

En resumen: a pesar de la baja de la titulación de anticuerpos obtenida con la cortisona, se presentó anemia hemolítica, con muerte del niño, dos horas después del nacimiento, a pesar de haberle practicado transfusión y demás cuidados que el caso requería.

Los autores recuerdan que las contraindicaciones médicas de la cortisona se delen tener en cuenta; la tuberculosis, la úlcera péptica, la colitis ulcerosa, la diaberes, las psicosis e hipertensión son contraindicaciones formales para su cinpleo.

M. A. F. B.

I)( )INTGENTERAPIA EN LA AMENORREA.-Doctores Alberto RAFinfle, Gullermo di Paola y Miguel Lelio.- Ia Semana Médica. Año I.X, encro 8, 1953.

Comentan los autores en su trabajo que a pesar de haber transcurrido casi uarenta anos desde que Van de Velde empleara la radioterapia en los trastornos W. ciclo, aún no es bien conocido el mecanismo por el cual actúan. Se discuten I. des teorias de estimulación o destrucción selectiva en los ovarios.

Los autores se adhieren a la segunda teoría por 3 razones: a) Reconocida acion destructora de los rayos $X$ sobre las diferentes estructuras ováricas. b) Iracaso de la radioterapia en las amenorreas graves; y c) Exito de la resección prircial de los ovarios.

Relatan además que este procedimiento se halla muy difundido en Bs. As. debidor a los buenos resultados obtenidos y a la sencillez del método. Sin embargo creen que esta medida terapéutica de gran valor no puede generalizarse a cualquicr tipo de amenorrea y su indicación debe ser cuidadosamente consideratla despues de los fracasos de los demás tratamientos hormonales.

Presentan una casuística de 41 casos tratados, de los cuales 21 fueron favorables y 20 resultaron negativos. De los 41 casos, 28 eran amenorreas leves (cfecto progesterona positivo) y curaron 20 o sea un $70 \%$. De las 13 restantes que cran amenorreas graves (efecto progesterona negativo) un solo caso curó o wea el $7 \%$. Anotan que de las 20 curadas con amenorrea leve el $40 \%$ presentaron embarazo ulterior. 
$\mathrm{La}$ indican exclusivamente en las amenorreas funcionales leves hipofiso: gonadales, es decir cuando los ciclos monofásicos cortos son el substratum fisiológico de la supresión del ciclo sexual. Estos casos se caracterizan por un extendido vaginal trófico, prueba de la progesterona positiva, endometrio proliferativo y cristalización del mocó cervical.

La contraindican: a) En las amenorreas leves por causa general, ya sea por factores ambientales, nutricionales o cmocionales. b) Amenorreas funcionales graves caracterizadas por la atrofia del aparato genital, extendido vaginal atrófico o hipotrófico, prucba de la progesterona negatival, endometrio atrofico y falta de cristalización del moco cervical. c) Amenorreas orgánicas ya sean hipofisarias, ováricas o uterinas. d) Despué de los trcinta y cinco años.

Como técnica usan en la amenorrea ovárica $100 \mathrm{r}$. medidas en el aire sobre cada ovario, en una o dos secciones. En las hipofisarias a más de la irradiación sobre ovarios le agregan 240 r. sobre cada campo lateral hipofisario. Las irradiaciones sobre ovarios pueden repetirse a los tres o cuatro meses.

Prohiben finalmente la relación sexual durante el tratamiento y durante un mes más por la posibilidad remota en las amenorreas, de irradiar un óvulo recientemente fecundado.

N. de la R.-Estamos en todo de acuerdo con el procedimiento a seguir en el tratamiento de la amenorrea leve hipofiso-gonadal con los rayos $\mathrm{X}$ y recalcamos que nunca se deben olvidar los procedimientos, para llegar a tal diagnóstico ni los tratamientos hormonales previos que con su fracaso nos dan mayores razones para el uso de este tratamiento.

\section{A. E. y E.}

\section{DIAGNOSTICO PRECOZ DE LA GESTACION POR EL MOCO CER. VICAL.-Doctores Gullerrmo di Paola, Miguel Lelo y Laura Eli- zeche.-La Prensa Médica Argentina. Vol. XI, No. 4, encro, 53.}

$\mathrm{Al}$ considerar las variaciones cíclicas del moco cervical en el ciclo bifásico los autores hacen un recuento histórico desde que Rydberg demostró la cristalización del mismo en forma de hojas de helecho. Llegan luego a la demostración de Lindestron de que este fenómeno es producido por cristalización del cloruro de sodio en presencia de mucina.

Los autores han estudiado la cristalización del cloruro de sodio en diversas soluciones hallando que la más próxima a la del moco cervical es del $0.9 \%$, concentración muy semejante a la del moco que es de $0.85 \%$. Han comprobado además los autores que en frotis negativos al agregarles solución de cloruro de sodio al $0.9 \%$ se producía una magnífica cristalización en hojas de helecho.

Relatan lo sostenido por Campos da Paz de que si existe cristalización segu: ramente la inseminación será normal y si no aquella será negativa de hecho.

Terminan su introducción citando el trabajo de Roland en el cual después de examinar 300 embarazadas entre dos dias y siete meses de su primera falla menstrual, no halló en ningún caso antes de los tres meses, cristalización típica positiva. 
A continuación describen lo técnica empleada en is treinta casos de mujeres grávidas entre los 35 y 90 días después de última menstruación. El diagnóstico fue corroborado por la R. de Galli y la evolución ulterior. En algunas, además, se practicó la prueba de la progesterona y en otras se controló la temperatura basal.

En 28 casos se comprobó la ausencia de cristalización típica $(++)$ y silo en dos casos se hallaron formas atípicas en pacientes de sesenta y sesenta i cinco dias de retardo menstrual, corroborando lo conocido de que la cristaliz.ción tipica sólo se observa: a) En la primera mitad del ciclo bifásico, entre lin siete y diecisćs días, siendo especialmente notable entre el undécimo y dé( momosexto dia (periodo ovulatorio). b) En todo ciclo monofásico (breve, me(di.no y prolongado). c) Durante la estrogenoterapia.

En su discusión recalcan sobre la importancia de la combinación cloruro de sodio-mucina la cual a su vez estaría supeditada a la acción estrogénica del ovario o la administración terapéutica de estrógenos.

La importancia de su trabajo la orientan más hacia aquellos transtornos menstruales en pacientes multíparas en que el embarazo no es esperado con gran complacencia $n$ en las cuarentonas donde frecuentemente comienzan a presentarse ciclos monofásicos. En estos casos el estudio del moco cervical es definitivo y de gran importancia.

No se debe olvidar la presencia de endocervicitis que alteran el resultado d.undo negatividad en ciclos monofásicos francos por acción de leucocitos y piocitos que impiden la cristalización.

N. de la R.-Parece que el estudio del moco cervical cada día adquiere mayor importancia clínica y los autores concuerdan en que da un alto margen de seguridad. En nuestro medio se hallan en periodo de estudio y todavia no tenemos bases para concluir tan categóricamente como los autores conocidos pero debido a su facilidad de ejecución y a su importancia diagnóstica es procedimiento que recomendamos para estudio y comprobaciones ulteriores.

$$
\text { A. E. y E. }
$$

\section{A AMENAZA DE ABORTO.-Arthun K. Koff y Alex Tulsky.-The} Surgical Clinics of North America. 1953. 33. 3.

La clínica enseña que el aborto es más frecuente antes de la duodécima semana del embarazo. Las causas productoras del aborto varían de acuerdo con la edad de la gestación. De una manera general se puede decir que el aborto es rvitable cuando únicamente se encuentra el síntoma hemorragia, una vez que x presentan los dolores y aparecen modificaciones en el cuello el aborto es inevitible.

Según algunos autores la causa de la hemorragia es la necrosis de la decidua la cual es a su vez consecuencia de una deficiente producción de progestetona por parte del corion placentario y del cuerpo lúteo. Ruthenford describe las siguientes lesiones anatomopatológicas de la decidua al cabo de 48 horas de extirpado el cuerpo lúteo: necrosis difusa y focal, trombosis, congestión de los sinusoides, hemorragias en el estroma y marcada infiltración polimorfonuclear. 
Los autores practicaron resecciones del cuerpo lúteo en pacientes a quienes se les iba a practicar el aborto terapéutico y la esterilización, al mismo tiempo se hicieron dosificaciones de pregnadiol antes, durante y después de la resección del cuerpo lúteo. Solamente tres de las ocho enfermas estudiadas presentaron sintomas de amenaza de aborto.

La frecuencia de la amenaza de aborto fluctúa del 10 al 20\%; de estos casos un $50 \%$ termina en aborto. El $86 \%$ de los abortos se presentan antes de la duodécima semana.

Las causas etiológicas del aborto se deducen del estudio anatomopatológico. Las alteraciones se pueden encontrar en el embrión, la placenta y las membranas. Hertig sostiene que en el $69.9 \%$ de los casos de aborto se encuentran degeneraciones hidatidiformes de la placenta. Mall y Streeter sostienen que solamente un $20 \%$ de los huevos abortados en el primer mes son normales, un $50 \%$ de los abortados en el segundo mes, y por el contrario los huevos abortados en el tercero y cuarto mes son normales en un $90 \%$, es decir, entre más joven sea el aborto mayores cambios anatomopatológicos.

Los principales factores que pueden afectar al huevo fertilizado son los siguientes:

19--Mala nutrición de la paciente.

2?-Anomalías en el aparato genital femenino, tales como fibromas, desviaciones uterinas, laceraciones del cuello, infecciones, etc.

39-Alteraciones en la producción de hormonas por parte del cuerpo lúteo, la placenta, el tiroides y las suprarrenales, que pueden conducir a una defectuosa formación de la decidua.

49-Infecciones crónicas de la madre, tales como nefritis. Se han producido alteraciones en el huevo fecundado provocando anoxia prolongada en el útero grávido. Las alteraciones de las células germinales son también muy importantes como causa etiológica en la producción de abortos.

Se acepta que el cuerpo lúteo produce progesterona y estrógenos a partir de las 48 horas de la ovulación y esta producción dura de 12 a 14 días, al cabo de los cuales el cuerpo lúteo cesa su función si no ha sobrevenido el embarazo. La implantación del huevo fertilizado ocurre al sexto o séptimo día de la ovulación y casi inmediatamente, a las 24 horas aparecen las gonadotrofinas coriónicas en la orina. La producción de esta hormona por parte del trofoblasto tiene por fin sostener y aumentar la actividad del cuerpo lúteo en su producción de progesterona y estrógeno. La producción de gonadotrofina coriónica llega a su máximo alrededor del quincuagésimo día del embarazo. Se sabe también que la producción de progesterona por parte de la placenta se inicia más o menos al trigésimo quinto día de la gestación, de tal manera que el cuerpo lúteo tiene acción, por medio de sus hormonas, en la implantación y sostenimiento del huevo fertilizado en los primeros días del embarazo, pero una vez que la placenta inicia la producción de hormonas pierde su importancia. Esto se demuestra fácilmente con el hecho conocido de que el embarazo continúa a pesar de la ex. tirpación del cuerpo lúteo. Es necesario aclarar que la progesterona y los estró 
genos son indispensables para la conservación del embarazo, pero su principal fucnte de producción está en la placenta.

De los estudios sobre el pregnandiol se han establecido los siguientes hechos:

19-El pregnandiol es un esteroide biológicamente inerte, producto de excreción de la progesterona.

2"-La excreción de pregnandiol por la orina en 24 horas es aproximadamente de 10 mgrs. durante la fase secretora del ciclo menstrual y durante los primcros sesenta dias del embarazo normal; de allí en adelante esa cantidad fluctúa entre 40 y 100 mgrs. hasta el octavo mes. No existe eliminación de pregnandiol durante la fase pre-ovulatoria del ciclo menstrual.

$3^{\circ}-\mathrm{Si}$ se inyecta progesterona durante la fase secretora del ciclo o en los primeros días del embarazo, solamente un $30 \%$ de esta sustancia se obtiene en la orina en forma de pregnandiol en las primeras 24 horas.

Muchos autores sostienen que el $80 \%$ de las amenazas de aborto se pueden salvar mediante el uso de la progesterona; pero existen otros como Colvin quien presenta un estudio de 1.500 casos en los cuales no se usó terapéutica endocrina y obtuvo un $70 \%$ de curaciones.

Guterman y Tulsky en un estudio sobre 125 pacientes con amenaza de ahrorto, establecen que cuando la eliminación de pregnandiol urinario es inferiur a 5 mgrs. en las 24 horas el pronóstico es muy malo y se presentará el aborto. en el $97 \%$ de los casos; en cambio cuando la eliminación de pregnandiol es de 10 mgrs. o más, el pronóstico mejora considerablemente. Estos autores aconscjan practicar rutinariamente las determinaciones de pregnandiol para estable cer al pronóstico y la terapéutica. Los resultados obtenidos empleando dosis de 5 a 25 mgrs. de progesterona son sensiblemente iguales a los obtenidos sin el empleo de esta hormona, tanto en los casos que presentan eliminación baja como en los que presentan eliminación normal de pregnandiol. Los autores emplearon dosis de 100 mgrs. diarios de progesterona en los casos en los cuales la rliminación de pregnandiol era inferior a $10 \mathrm{mgrs}$ y con esta dosis obtuvieron wraciones en un $30 \%$, en cambio en casos similares en los cuales emplearon desis de 5 a 25 mgrs. solamente obtuvieron 3\% de curaciones.

Se establecen las siguientes conclusiones:

1.-El cuerpo lúteo no es esencial en el mantenimiento del embarazo en la mujer.

2.-La progesterona no solamente es producida por el cuerpo lúteo sino también por la placenta.

3.-Los niveles de pregnandiol son índices de la excreción de la progesterona.

4,-Niveles normales de pregnandiol son de buen pronóstico en la amenaza del aborto.

5.-Niveles bajos en la eliminación de pregnandiol son de mal pronóstico en la amenaza de aborto. 
6.-Se puede decir que dosis de 5 a 25 mgrs. de progesterona no mejoran los resultados en el tratamiento de la amenaza de aborto.

7.-La dosis de 100 mgrs. diarios sostenida hasta que cesen los síntomas de la amenaza de aborto pueden ser muy útiles en el tratamiento cuando existe una deficiencia de progesterona, demostrada por una baja eli. minación de pregnandiol.

\section{J. A. R.}

EL USO DEL PITOCIN ENDONENOSO EN EL PARTO. Informe pre. sentado sobre 100 casos.-Raymond 11. Katilman, M. D. Stanley M. M. D., and Wu.fred J. Ratzın, M. D.-New York. Am. J. Obst. Ginec. February, 1953.

Experiencias vcrificadas en el Beth Israel Hospital en 100 enfermas observadas consecutivainente: 50 nuliparas y 50 multíparas (para i: 40; para ii: 8; para iii: 2 casos)

Diluciones usadas: desde 2 hasta $71 \frac{1}{2}$ minimas de Pitocín en 500 c. c. de solvente (glucosa al $5 \%$ en agua o en solución salina).

Los casos fueron debidamente estudiados, descartando desproporciones cefalopélvicas por el examen clínico y por radiopelvimetría.

Las indicaciones fueron:

A.-Grupo de inducción: Inducción electiva: 23 casos, ruptura de membrana: 9 casos, preeclampsia: 5 casos, indicación facultativa: 5 casos, embarazo prolongado: 3 casos, prediabetes: 1 caso.

B.-Grupo en que se empleó después de iniciado el trabajo: uso electivo: 24 casos, inercia uterina primitiva: 18 casos, inercia uterina secundaria: 9 casos, cuello espasmódico: 2 casos, preeclampsia: 1 caso.

Las conclusiones son las siguientes:

1.-La administración intravenosa de Pitocín en solución es un medio se guro y eficiente para estimular el trabajo del parto, cuanto éste es debidamente controlado.

2.-Su uso da excelente resultado en el tratamiento de la inercia uterina primitiva y secundaria.

3.-El Pitocín intravenoso usado en pacientes en trabajo, acorta considerablemente el promedio de duración de éste.

4.-El uso del Pitocín intravenoso, asociado a anestesia en silla se sugiere como posible medio de tratamiento en los casos de cuello espasmódico.

5.-El Pitocín endovenoso es un medio eficiente para inducir al parto. Dos son las condiciones más importantes para su éxito: cuello blando y membranas rotas. En casos debidamente seleccionados, el fracaso de la inducción es raro, y al insistir sólo un bajo porcentaje inicia trabajo.

6.-El Pitocín endovenoso puede dejarse que continúe hasta después del parto; el no hacerlo, aumenta la incidencia de la atonía uterina post-partum.

7.-El Pitocín endovenoso usado por un largo período durante el parto, no tiene efecto ninguno sobre la tensión arterial y el pulso de la madre. 
8.-El uso continuado de P.t cén endovenoso en casos de inercia uterina que no han respondido, está contraindicado y es peligroso.

\section{A. F.}

\section{ISTADO ACTUAL DE LA OPERACION CESAREA.-]. P. Greenhill.} The Surgical Clinies of North América. 1953. 33. 87.

En este artículo el autor hace un estudio bastante completo acerca de la oferación cesárea, sus indicaciones, sus contraindicaciones y un análisis estadístho basido en los datos obtenidos en el Chicago Lying-in Hospital.

El número de operaciones cesáreas aumenta cada ano en los Estados Unidx y este aumento no está en relación con el aumento en el número de nacimicntos. El aumento de nacimientos por vía abdominal se debe a un aumento de las indicaciones de la cesárea.

En la práctica es difícil enumerar las indicaciones de la cesárea, puesto que esta conducta se ha aplicado a casi todas las complicaciones obstétricas. El autor puntualiza las siguientes indicaciones:

1.-Desproporción comprobada entre el estrecho superior y la cabeza fetal. Esta desproporción no puede ser definitivamente aceptada sino después de una prucba de trabajo. Los datos clínices y radiológicos no son definitivos en $\mathrm{mu}$ hor casos. La prueba del trabajo se debe sistematizar y se aconseja lo siguiente: drypués de que la enferma llega al parto espontáneamente se debe dejar 18 lioris en trabajo regular. En caso de ruptura prematura de las membranas la duración de la prueba del trabajo debe limitarse a unas 6 horas.

2.-Disminución COMPROBADA DE LOS DIÁMETROS DEL ESTRECHO INFEแแm. En los casos en los cuales el diámetro bi-isquiático sea inferior a 6 cmts. " que la suma del diámetro bi-isquiático y el sagital posterior sea inferior a 15 comts debe hacerse la operación cesárea. El diagnóstico de estrechez a este nivel debe hacerse siempre antes de iniciado el parto.

3.-Disminución acentuada de la escavación pélvica.

4.- Cesárea previa. En estos casos la indicación es absoluta si persisten las undiciones que determinaron la cesárea anterior. El antiguo lema "una vez ces.rua siempre cesárea", debe tenerse muy presente debido a que el peligro de ruptura uterina es mayor en estos casos. A las pacientes que presenten el antecedente de cesárea e inicien un trabajo regular seguido de rápida dilatación del cuello y descenso de la cabeza fetal se les deberá aplicar sistemáticamente un forceps de desprendimiento y hacer una revisión de la cavidad uterina después del parto.

5.-Tumores previos que obstruyen el canal genital.

6.- Pre-eclampsia en primigrávida con feto viable que no ha respondidá a un tratamiento médico bien dirigido.

7.--Placenta previa central total y central parcial y algunos casos de otras variedades en las cuales la hemorragia es severa.

8.-Abruptio placenta con hemorragia intensa y aun con feto muerto.

9.--Situaciones transversas en primigrávida en las cuales ha fallado la versión por maniobras externas. 